\title{
Rotational dynamics of a particle in a turbulent stream
}

\author{
Yayun Wang* and Adam Sierakowski ${ }^{\dagger}$ \\ Department of Mechanical Engineering, Johns Hopkins University, 3400 North Charles Street, \\ Baltimore, Maryland 21218, USA \\ Andrea Prosperetti \\ Department of Mechanical Engineering, University of Houston, 4726 Calhoun Rd, \\ Houston, Texas 77204-4006, USA \\ and Faculty of Science and Technology and J.M. Burgers Centre for Fluid Dynamics, \\ University of Twente, P.O. Box 217, 7500 AE Enschede, The Netherlands
}

(Received 30 November 2017; published 19 June 2019)

\begin{abstract}
The paper presents results for the resolved numerical simulation of a turbulent flow past a homogeneous sphere and a spherical shell of equal mass and radius (and, therefore, with a larger moment of inertia) free to rotate around a fixed center. This situation approximates the behavior of a particle whose relative motion with respect to the fluid is driven by external forces, such as a density difference in a gravitational field. Holding the center fixed makes it possible to have precise information on the turbulent flow incident on the particle by repeating the same simulations without the particle. Two particle Reynolds numbers based on the mean velocity, $\operatorname{Re}_{p}=80$ and 150 , are investigated; the incident turbulence has $\operatorname{Re}_{\lambda}=36$ and 31, respectively. The particle diameter is an order of magnitude larger than the Kolmogorov length scale and close to the integral length scale. The turbulent eddies that interact most strongly with the particle are characterized. Their size is found to increase with $\operatorname{Re}_{p}$ due to the interplay of the convection timescale, the particle timescale, and the eddy timescale, but it remains of the order of the particle diameter. The sign of the hydrodynamic torque is likely to persist much less than the convection time, although longer durations are also found, revealing the effect of occasional interactions with larger eddies. The autocorrelation of the torque changes sign at shorter and shorter fractions of the convection time as the Reynolds number increases. Significant cross-stream forces are found. An analysis of their magnitude shows that they are mostly due to induced vortex shedding combined with a weaker Magnus-like mechanism.
\end{abstract}

DOI: 10.1103/PhysRevFluids.4.064304

\section{INTRODUCTION}

The development of new experimental techniques [see, e.g., 1-9] and numerical methods [see, e.g., 10-15] is making it possible to study the interaction of turbulence with particles significantly larger than the Kolmogorov scale for which rotation cannot be disregarded and the earlier pointparticle models [see, e.g., 16,17] are inappropriate. Much of this work has dealt with aspects of the translational motion of particles, such as the statistics of particle velocity and acceleration [2]. Less attention has been paid to particle rotation. The rotational intermittency and lift experienced

\footnotetext{
*WangYayun1990@gmail.com; www.physaliscfd.org

†sierakowski@jhu.edu

$\doteqdot$ aprosper@central.uh.edu
} 
by a spherical, neutrally buoyant particle in homogeneous turbulence were studied in Refs. [1,2]. The particle size was comparable to the integral scale and the particle Reynolds numbers were of the order of 1000 . The results showed a strong intermittency of the angular dynamics, with the probability density function (PDF) of the angular acceleration having a flatness of about 7 , considerably larger than that of the angular velocity, which was close to 4 . The root-mean-square (RMS) angular acceleration was found to be of the order of $\left(u^{\prime} / 2 a\right)^{2}$, with $u^{\prime}$ the RMS of the turbulent velocity fluctuations and $a$ the particle radius. The authors write, "Which properties of the turbulent flow control the rate of rotation of the particle also remains to be elucidated... Small eddies acting on the particle in a spatially incoherent manner would result in a significantly reduced torque acting on the particle. This suggests a much more coherent flow pattern, in fact consistent with the recent numerical results of" [18].

The extent of the fluid region most influencing the motion of neutrally buoyant spheres in homogeneous turbulence was studied in Ref. [3]. The conclusion was that flow structures somewhat larger than the particle diameter interact most strongly with it. A similar result has been found in studies directed primarily to the investigation of the effects of particle shape, including ellipsoids, disks and rods [4,5]. While spherical particles were found to have a larger effect on the fluid turbulence than prolate ellipsoids, the auto-covariances of ellipsoids and spheres were statistically identical. From this observation the authors conclude that rotation is controlled by turbulent scales larger than the particle size. The results of Ref. [8] and [9] show that the rotation statistics of particles of various shapes is most sensitive to the volume-equivalent spherical diameter, which again leads to similar conclusions. A qualitatively similar result is reported in [19] for the rotational dynamics of neutrally buoyant rods. For all rod lengths, the correlation time of the Lagrangian autocorrelation of the rotation rate scales as the turn-over time of eddies of the size of the rod.

In the studies mentioned so far neutrally or nearly neutrally buoyant particles were used. More recently, particles with a density significantly smaller than the surrounding liquid were used in Refs. [6,7]. This difference caused a larger particle-liquid relative velocity with a Galilei number in the range 30-3000 and the development of a wake and vortex shedding. As a consequence, unlike the equal-density case, both velocity and acceleration de-correlate at the same rate, which is explained by the determining influence of vortices shed in the wake.

In this paper, as a step toward a better understanding of the "properties of the turbulent flow (which) control the rate of rotation of the particle" [2], we use fully resolved numerical simulations to study the rotational dynamics of a single spherical particle free to rotate around its center held fixed in an decaying incident turbulent stream at two Reynolds numbers, $\operatorname{Re}_{p}=80$ and 150. By keeping the particle center fixed, and comparing with the identical incident flow in the absence of the particle, we can relate the particle rotational motion to features of the incident turbulence.

Our interest lies in particles with a size close to the integral scale, two or three times larger than the Taylor microscale and over a factor of 20 larger than the Kolmogorov scale. For such particles, which have a size comparable to that of the objects used in the experiments mentioned before, rotational inertia is important. The consideration of moments of inertia in the ratio 1:5/3 provides further insight into the role of inertia. The fact that the fluid flow has a nonzero mean velocity and that the particle is allowed to rotate are the main differences between the present work and our earlier study [18], in which the particle was held fixed in a homogeneous isotropic turbulent field.

A point to stress is that the numerical method used in this work is singularly appropriate for this problem as it leads to a very accurate evaluation of the hydrodynamic torque on the particle, as documented in Ref. [20].

The forced stationarity of the particle center approximates the buoyant relative motion studied in Refs. [6,7]. As in that paper, we find significant effects of vortex shedding induced by the turbulence transported by the mean flow. We present some evidence suggesting the presence of Magnuslike forces which, however, are found to play a lesser role compared with those due to vortex shedding. 
TABLE I. Simulation parameters at the inlet plane and at the plane containing the particle center for $\operatorname{Re}_{p}=$ 80 (upper two lines) and 150; $d$ is the particle diameter; $\eta=\left(v^{3} / \epsilon\right)^{1 / 4}$ the Kolmogorov length scale, $\lambda_{g}$ the Taylor length scale, $L=u^{\prime 3} / \epsilon$ the integral length scale, $u^{\prime}$ the RMS of velocity fluctuations and $\Delta x$ the mesh length.

\begin{tabular}{lccccccc}
\hline \hline $\operatorname{Re}_{p}$ & & $\operatorname{Re}_{\lambda}$ & $\eta / d$ & $\lambda_{g} / d$ & $L / d$ & $u^{\prime} / U$ & $\eta / \Delta x$ \\
\hline 80 & Inlet & 59.1 & 0.0223 & 0.337 & 1.33 & - & 0.668 \\
& Particle center & 36.1 & 0.0366 & 0.431 & 1.06 & 1.04 & 1.10 \\
\hline 150 & Inlet & 47.2 & 0.0313 & 0.423 & 1.33 & - & 0.938 \\
& Particle center & 30.7 & 0.0454 & 0.495 & 1.03 & 0.413 & 1.36 \\
\hline \hline
\end{tabular}

\section{NUMERICAL METHOD}

The simulations are performed with the Physalis method, a complete description of which is available in several papers including, most recently, Ref. [21]; implementation details are described in Ref. [22]. The Navier-Stokes equations are solved on a fixed Cartesian grid by a projection method. A characteristic feature of the method is the way in which the fluid is coupled to the particle, assumed to have a no-slip spherical surface. The coupling is based on the recognition that, in the vicinity of the particle surfaces, the fluid motion differs little from a rigid-body motion. This circumstance permits the Navier-Stokes equations to be linearized to the Stokes form, for which the general exact solution, obtained by Lamb [23,24], is available. This analytical solution is used as a "bridge" between the particle surface and the closest nodes of the Cartesian grid thus bypassing the difficulties deriving from the complex geometrical relationship between the spherical particles and the underlying Cartesian grid. The particle orientation is updated on the basis of the calculated hydrodynamic torque $\mathbf{L}$ :

$$
I \frac{d \mathbf{\Omega}}{d t}=\mathbf{L},
$$

in which $I$ is the particle moment of inertia and $\boldsymbol{\Omega}$ is the particle angular velocity.

The method, which has been extensively validated in earlier papers [see, e.g., 20,21], is accurate and efficient. Since the Lamb solution is expressed as a series of spherical harmonics, the error decreases exponentially, rather than algebraically, as the number of degrees of freedom used to describe each particle is increased. This feature is in marked contrast with the algebraic error decrease of most other methods, such as the immersed-boundary method. The no-slip condition at the particle surface is satisfied exactly for any degree of truncation of the series expansion. A unique feature of Physalis, which makes it very suitable for the present study, is that the coefficients of the expansion directly furnish the torque acting on the particle with no need for additional calculations. For these reasons the method furnishes the torque on the particle with a very high degree of accuracy which would be difficult to approach with conventional immersed-boundary methods. In the present work, the Lamb expansion was truncated keeping terms of order 0, 1, and 2, which corresponds to retaining a total of 25 coefficients as in Ref. [25].

Isotropic, homogeneous turbulence is generated in a $210 \times 210 \times 210$-cells cubic domain using the linear forcing scheme of Refs. [26-28] (see Fig. 1). We checked that the features of the turbulence, and in particular the intensity and integral length scales, matched the results reported in Ref. [27]. This turbulent field, augmented by a constant velocity $U$ along the $z$ direction, is imposed at the inlet of an equal domain containing the particle in the manner described in Ref. [25]. The integral timescale, defined below in Eq. (2), is at least four times shorter than the convection time over the length of the turbulence-generating domain, which ensures the absence of artificial periodicity in the convected turbulence as discussed in [25]. As shown in Table I, the turbulence undergoes a strong decay as it is transported toward the particle. 


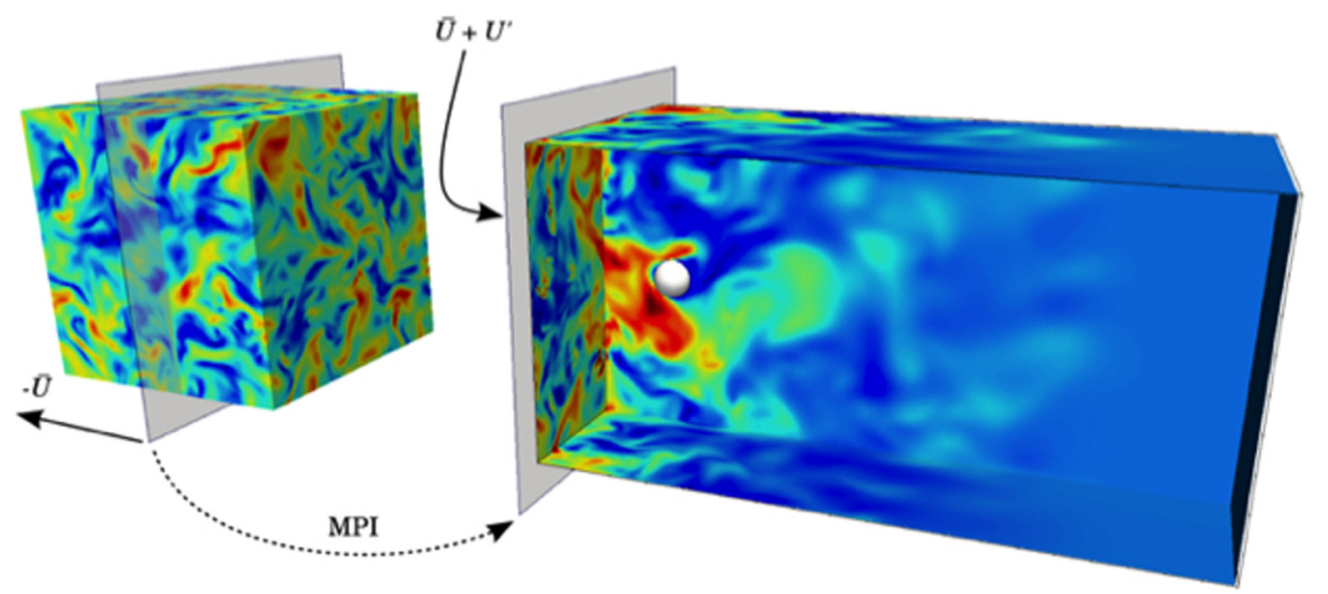

FIG. 1. Schematic illustration of how the turbulence affecting the particle is generated. Zero-mean-velocity homogeneous isotropic turbulence is generated in the cubic domain on the left. The velocity field seen by a plane moving to the left in the cubic domain with velocity $U$ is augmented by a constant velocity $U$ along the long dimension and used as inlet flow velocity for the longer domain containing the particle; see Ref. [25] for details.

We use 30 mesh lengths $\Delta x$ per particle diameter $d$ which, on the basis of our previous experience, provides a very good accuracy in the range of Reynolds numbers relevant for this study. The sides of both domains in the cross-stream direction have a length of $7 d$ so that the area blockage due to the particle is less than $2 \%$. We consider two different particle Reynolds numbers $\operatorname{Re}_{p}=d U / v$ (with $v$ the kinematic viscosity of the fluid), $\operatorname{Re}_{p}=80$ and 150 . The particle center is fixed at a distance $3.5 d$ downstream of the inlet boundary in a symmetric position with respect to the lateral boundaries of the domain. The turbulence decays as it is convected toward the particle, and the forcing is adjusted so that, at the plane of the particle center, the values of the Taylor microscale Reynolds number, $\operatorname{Re}_{\lambda}$, are comparable, 36 for $\operatorname{Re}_{p}=80$ and 31 for $\operatorname{Re}_{p}=150$.

\section{PARAMETER VALUES}

Values of the parameters characterizing the incident flow are shown in Table I. By the time the turbulence has reached the plane of the particle center, the Taylor microscale is about $1 / 3-1 / 2$ of the particle diameter, while the Kolmogorov length $\eta=\left(v^{3} / \epsilon\right)^{1 / 4}$ is more than one order of magnitude smaller. From the last column of the table, showing values of $\eta / \Delta x$, it can be seen that the Kolmogorov scale is adequately resolved [see, e.g., 29, p. 347].

Several timescales are relevant for the present problem. In the first place, the turbulence is characterized by the Kolmogorov timescale $\tau_{K}$ and the integral timescale $\tau_{E}$, respectively given by

$$
\tau_{K}=\sqrt{\frac{\nu}{\epsilon}}, \quad \tau_{E}=\frac{k}{\epsilon}
$$

with $k$ the turbulent kinetic energy and $\epsilon$ the dissipation, both evaluated at the particle plane. The particle response time for rotational motion is given by

$$
\tau_{p}=\frac{I}{8 \pi \mu d^{3}}
$$

where $\mu$ is the fluid viscosity. On the basis of these three timescales we can define two Stokes numbers:

$$
\mathrm{St}_{K}=\frac{\tau_{p}}{\tau_{K}} \quad \text { and } \quad \mathrm{St}_{E}=\frac{\tau_{p}}{\tau_{E}} .
$$


TABLE II. Numerical values of several quantities characterizing the simulations; $\tau_{c}=d / U$ is the convection time past the particle, $\tau_{E}$ the integral timescale, $\mathrm{St}_{K}$ and $\mathrm{St}_{E}$ the Stokes numbers based on the Kolmogorov and integral timescales; $O_{\mathrm{fl}}$, defined in Eq. (5) is the mean angular velocity of a fluid eddy with the size of the particle; $\ell_{p} / d$ is the size of eddies having the same time constant $\tau_{p}$ as the particle. The particle timescale shown here has been calculated for the solid sphere; that for the shell is a factor 10/3 larger.

\begin{tabular}{lcccccccc}
\hline \hline $\operatorname{Re}_{p}$ & $\tau_{c} / \tau_{E}$ & $\tau_{c} / \tau_{p}$ & $\mathrm{St}_{K}$ & $\mathrm{St}_{E}$ & $\tau_{E} O_{\mathrm{fl}}$ & $\tau_{p} O_{\mathrm{fl}}$ & $\nu \tau_{E} / d^{2}$ & $\ell_{p} / d$ \\
\hline 80 & 0.669 & 0.376 & 25.37 & 1.78 & 0.778 & 1.38 & 0.0187 & 4.59 \\
150 & 0.271 & 0.152 & 16.33 & 1.78 & 0.763 & 1.36 & 0.0246 & 4.49 \\
\hline \hline
\end{tabular}

Numerical values of these and other normalized scales to be introduced presently are provided in Table II.

In spite of the fact that our particle size is close to the integral length scale, inertial-range scaling provides a convenient, if approximate, framework to analyze the particle-turbulence interaction as confirmed by the fact that the scaled mean angular velocities and hydrodynamic torques are numbers of order 1 (see Table III below). On this basis, we normalize the particle angular velocity by the angular velocity of an eddy of the same size of the particle which, for homogeneous isotropic turbulence, can be estimated to be

$$
O_{\mathrm{fl}}=\frac{1}{2}\left(\frac{\epsilon}{d^{2}}\right)^{1 / 3}
$$

where the factor $\frac{1}{2}$ reflects the relation between the vorticity and the angular velocity of a fluid particle.

We define a dimensionless moment of inertia $I^{*}$ by

$$
I^{*}=\frac{I}{\frac{1}{10}\left(\frac{\pi}{6} d^{3} \rho\right) d^{2}},
$$

with $\rho$ the fluid density. We consider two different values of this quantity, $I^{*}=2$ and $10 / 3$, the former corresponding, for example, to a particle with a uniform density $\rho_{p} / \rho=2$, the latter to a thin spherical shell with the same radius and mass as the particle. The particle timescale Eq. (3) is, in terms of $I^{*}, \tau_{p}=\left(I^{*} / 480\right) d^{2} / \nu$.

The length scale $\ell_{p}$ of eddies having the same timescale as the particle is readily estimated by equating the timescale $\tau_{\ell}$ of an inertial-range eddy of size $\ell$, as given by the theory of homogeneous isotropic turbulence,

$$
\tau_{\ell}=\left(\frac{\ell^{2}}{\epsilon}\right)^{1 / 3}
$$

TABLE III. Root-mean-square values of the normalized angular velocity and torque components.

\begin{tabular}{llllr}
\hline \hline & \multicolumn{2}{c}{$\operatorname{Re}_{p}=80$} & \multicolumn{2}{c}{$\operatorname{Re}_{p}=150$} \\
\cline { 2 - 3 } & sphere & shell & sphere & shell \\
\hline$\Omega_{x}^{* \prime}$ & 0.580 & 0.424 & 0.378 & 0.328 \\
$\Omega_{y}^{* \prime}$ & 0.492 & 0.438 & 0.356 & 0.329 \\
$\Omega_{z}^{* \prime}$ & 0.503 & 0.469 & 0.389 & 0.306 \\
$L_{x}^{* \prime}$ & 1.525 & 1.716 & 1.275 & 1.630 \\
$L_{y}^{* \prime}$ & 1.469 & 1.665 & 1.255 & 1.517 \\
$L_{z}^{* \prime}$ & 1.295 & 1.572 & 1.008 & 1.193 \\
\hline \hline
\end{tabular}


to $\tau_{p}$ given in Eq. (3) to find

$$
\frac{\ell_{p}}{d}=\left(8 \tau_{p} O_{\mathrm{fl}}\right)^{3 / 2}
$$

The values of $\ell_{p} / d$ produced by this relation are shown in Table II. For both values of $\operatorname{Re}_{p}$ we find $\ell_{p} / d \sim 4.5$, which is significantly larger than the integral length scale. Even if present, therefore, such long-lived eddies will be very infrequent in both situations investigated. Thus, since the particle will mostly interact with eddies having a smaller characteristic time than its own, one may expect that the angular velocity that it will pick up will be smaller than that of the eddies with which it interacts. Another factor reinforcing this expectation is that, as Table II shows, the convection time $\tau_{c}$ past the particle,

$$
\tau_{c}=\frac{d}{U}
$$

is also shorter than the timescale $\tau_{p}$ of the particle.

As shown in Table I, $u^{\prime} / U$ is about 1 for $\operatorname{Re}_{p}=80$, and about 0.4 for $\operatorname{Re}_{p}=150$. A Taylorhypothesis-like picture of turbulent eddies frozen in the flow is therefore inappropriate in the first case and not very well justified even in the second one. Nevertheless, it is of some interest to estimate the spatial scale $\ell_{c}$ of an eddy having the same timescale as the convection time Eq. (9) past the particle at the velocity of the imposed mean flow. Unless the eddy persisted at least this long, it would be unlikely that it be able to impart much rotation to the particle. Upon setting $\tau_{\ell}=\tau_{c}$ and solving for $\ell \equiv \ell_{c}$ we find

$$
\frac{\ell_{c}}{d}=\frac{1}{\operatorname{Re}_{p}^{3 / 2}}\left(\frac{d}{\eta}\right)^{2} .
$$

For $\operatorname{Re}_{p}=80$ and $\operatorname{Re}_{p}=150$ this relation gives $\ell_{c} / d \simeq 1.04$ and $\ell_{c} / d \simeq 0.264$, respectively. On average, eddies smaller than the scale indicated by this relation will not maintain their integrity for the time during which they are able to interact with the particle. As $\operatorname{Re}_{p}$ increases, this threshold becomes less stringent and more eddies will last long enough to interact with the particle. However, the effect of this trend is countered by the fact that smaller eddies have a smaller energy even though they carry a larger vorticity.

An eddy of size $\ell$ cannot impart to the particle a greater angular velocity than its own, of order $1 / \tau_{\ell}$, nor can it give the particle more kinetic energy that itself has, of the order of $\rho \ell^{3} \epsilon \tau_{\ell}$. By equating this energy to that of a particle rotating with the eddy's angular velocity $1 / \tau_{\ell}$ we can estimate the length scale of these hypothetical "asymptotic eddies" (in the sense that they transfer all their energy to the particle and give it the largest angular velocity that they can impart) to be $\ell / d \sim$ $\left(\pi I^{*} / 120\right)^{1 / 5} \simeq 0.55$ for $I_{*}=2$. The numerical constant is just a crude estimate as it will depend on the shape of the eddy, on whether the particle angular velocity is taken as $1 / \tau_{\ell}$ or $1 / \frac{1}{2} \tau_{\ell}$ and so on, but it nevertheless shows that an eddy too much smaller than the particle diameter cannot impart to the particle a significant angular velocity even if the interaction occurred under the most favorable idealized conditions. All of these considerations lead us to expect that the angular velocities picked up by the particle will be relatively small and unlikely to be larger (in modulus) than the scale $O_{\mathrm{fl}}$ defined in Eq. (5).

For each value of $\operatorname{Re}_{p}$ and each realization of the turbulent flow, three types of simulations were carried out, one without the particle, one with a particle with $I^{*}=2$, referred to as "solid sphere," and one with a spherical shell of equal radius and mass, having the larger moment of inertia $I^{*}=$ $10 / 3$. To mitigate the effect of statistical fluctuations, the results that we present have been obtained by averaging over seven different realizations of the turbulent field, each one lasting between 60 and 100 integral times as calculated in correspondence of the inlet conditions. Each set of simulations required about 12 weeks on one-half of an Nvidia K80 GPU (essentially equivalent to one K40) installed in a Dell PowerEdge R730 configured with dual Intel Haswell Xeon E5-2680v3 CPUs and two Nvidia K80 GPUs. 
For fixed, nonrotating spheres in steady uniform flow, experimental values of the drag coefficient $C_{D}=F_{z} /\left(\frac{\pi}{8} \rho d^{2} U^{2}\right)$ are 1.23 for $\operatorname{Re}_{p}=80$ and 0.894 for $\operatorname{Re}_{p}=150$ [30]. The well-known SchillerNaumann correlation [see, e.g., 31] gives 1.21 and 0.910, respectively. Drag coefficients in turbulent flow are larger [see, e.g., 32]. In our case, for $\operatorname{Re}_{p}=80$ we find $C_{D}=1.31 \pm 0.52$ while, for $\operatorname{Re}_{p}=$ $150, C_{D}=1.05 \pm 0.09$. These values have been calculated by averaging over the last 40 integral times to avoid the effect of the initial transients. The large standard deviation for $\operatorname{Re}_{p}=80$ is due to the very strong turbulence intensity (see Table I). Due to the strong intermittency, convergence of the averaging is very slow in this case. Interestingly, the results for the spherical shell are different, $C_{D}=1.06 \pm 0.44$ and $1.19 \pm 0.15$ for $\operatorname{Re}_{p}=80$ and $\operatorname{Re}_{p}=150$, respectively. It may be observed that the average values for the solid sphere decrease with increasing $\operatorname{Re}_{p}$, as expected, while those for the shell seem to slightly increase. Whether this is a consequence of insufficient statistics or a genuine physical effect cannot be determined on the basis of the numerical results. Generally speaking, one would expect that, on increasing the moment of inertia, the particle should become more and more reluctant to rotate and, therefore, behave more and more like a fixed sphere. On this basis, we incline toward the first explanation.

\section{RESULTS: ANGULAR VELOCITY AND ACCELERATION}

We nondimensionalize the particle angular velocity in terms of $O_{\mathrm{fl}}$, the angular velocity of eddies having the scale of the particle diameter, defined in Eq. (5):

$$
\boldsymbol{\Omega}^{*}=\frac{\boldsymbol{\Omega}}{O_{\mathrm{fl}}} \text {. }
$$

In the calculation of $O_{\mathrm{fl}}, \epsilon$ is evaluated on the plane of the particle center in the absence of the particle. The RMS values of the three components of this normalized angular velocity are shown in Table III.

The rotational Reynolds number for the particle is given by

$$
\operatorname{Re}_{\Omega}=\frac{d^{2} \Omega}{v}=\frac{1}{2}\left(\frac{d}{\eta}\right)^{4 / 3} \Omega^{*} .
$$

With the numerical values of Tables I and III this is of the order of 20. Inertial effects are therefore important, but it is convenient to nondimensionalize the torque on a viscous scale by

$$
\mathbf{L}^{*}=\frac{\mathbf{L}}{8 \pi \mu d^{3} O_{\mathrm{fl}}} .
$$

The RMS values of the three components of this normalized torque are also shown in Table III. The results for both $\boldsymbol{\Omega}^{*}$ and $\mathbf{L}^{*}$ are numbers of order one, which shows the adequacy of the nondimensionalizations adopted in spite of the magnitude of the rotational Reynolds number. For both quantities, the components in the cross-stream plane are comparable, as expected. The angular velocities for the sphere are somewhat larger than for the shell, which can be imputed to the smaller rotational inertia. For the same reason, the torques acting on the shell are larger than those acting on the sphere, in agreement with the results shown later. It appears likely that this feature is a consequence of the larger difference between the angular velocity of the the fluid and that of the shell which, due to its larger inertia, opposes a greater resistance to rotation. The torque in the flow direction is somewhat smaller than that in the cross-stream plane, which suggests some difference in the way in which the turbulent eddies responsible for rotation in the different directions interact with the particle.

It was suggested in Ref. [19] that the mean-square particle angular velocity $\langle\boldsymbol{\Omega} \cdot \boldsymbol{\Omega}\rangle$ satisfies a relation of the form

$$
\langle\boldsymbol{\Omega} \cdot \boldsymbol{\Omega}\rangle \tau_{K}^{2}\left(\frac{\hat{d}}{\eta}\right)^{4 / 3} \simeq 2 .
$$


The length $\hat{d}$ used in Ref. [19] was the length of their rods. A similar relation was also found to be verified in Refs. [8,9], where $\hat{d}$ was taken as the volume-equivalent spherical diameter of their particles. In terms of the dimensionless variables used here, the left-hand side of this relation is simply

$$
\frac{1}{4}\left[\left(\Omega_{x}^{* \prime}\right)^{2}+\left(\Omega_{y}^{* \prime}\right)^{2}+\left(\Omega_{z}^{* \prime}\right)^{2}\right]
$$

With the results shown in Tables I, II, and III for the sphere this quantity evaluates to 0.208 and 0.105 for $\operatorname{Re}_{p}=80$ and 150 , respectively. The order-of-magnitude difference with the reported experimental values is likely due to the fact that the particles used in the experiments were free to move and neutrally buoyant. Thus, they were able to interact with the turbulent eddies for a much longer time than the convection time $\tau_{c}$ for which our fixed particle remained exposed to them. The fact that the quantity Eq. (15) is smaller for the larger convection velocity (i.e., the larger particle Reynolds number and the shorter interaction time) lends some support to this conjecture. Of course, the much larger $\mathrm{Re}_{\lambda}$ in the experiment (about 250 versus the present one close to 30) may also play a role. Since Eq. (15) is independent of $d / \eta$, the validity of the scaling Eq. (14) would also imply a constant value of the dimensionless angular velocity Eq. (15) irrespective of the Reynolds number. The fact that our results do not support this consequence of Eq. (14), irrespective of the numerical value in the right-hand side, gives another indication of the difference between our situation and the experiments referred to.

Another interesting remark to be made in connection with the results of Table III is that the root-mean-square values of the angular velocities are larger for $\operatorname{Re}_{p}=80$ than $\operatorname{Re}_{p}=150$. This result is likely due to the fact that, for the same convected turbulence, a particle will be exposed to eddies having size and energy able to set it into rotation for a shorter and shorter time as the speed of the incident flow increases.

The two panels in Fig. 2 show the PDF of $\Omega_{x, y}^{*}$, the particle angular velocity in the plane perpendicular to the mean incident velocity. Since the directions $x$ and $y$ are equivalent, here and in the graphs that follow we have not distinguished between them in calculating the PDFs. The upper panel is for $\operatorname{Re}_{p}=80$ and the lower one for $\operatorname{Re}_{p}=150$. The tallest, most peaked curves (red) are for the shell and the somewhat lower and broader ones (black) for the solid sphere. The lowest curve (blue) is the PDF for the fluid angular velocity (i.e., vorticity/2) averaged over a sphere of radius $d / 2$ while the other one (yellow) is for the fluid angular velocity averaged over a sphere of radius $d$, both calculated at the particle position in the absence of the particle. The dashed lines are Gaussian fits. The RMS values are 3.7179 (for the blue curve) and 1.8361 (for the yellow curve). These values are larger than those for the particle shown in Table III, which is another indication of the inability of the smaller eddies to impart a significant angular velocity to the particle in spite of their larger angular velocity.

The PDF's for the solid sphere and the shell closely approximate Gaussian distributions with a flatness deviating by less than $5 \%$ from 3 . The PDF's for the angular velocity in the flow direction (not shown) are similar. The PDF's of the averaged fluid vorticity are much broader and exhibit intermittency with a flatness of about 4.2, although they appear to exhibit only a small difference from the corresponding Gaussian fits (dashed lines). These results agree with the observations reported in Ref. [8], which suggest that particles with $\eta / d<0.037$, in a turbulent field with $\operatorname{Re}_{\lambda} \simeq 250$, "do not respond, in any distinct way, to the intermittency of the surrounding turbulence." Reference [8], however, also reports that the particle angular velocity has a statistical distribution similar to that of the vorticity filtered over scales comparable with the particle diameter. This generates a paradox; citing from [8]: "This statistical analogy [the Gaussianity of the particle angular velocity and other results] suggests that inertial particles experience turbulence only as random forcing without responding to any structure of turbulence. However, it contradicts evidences that these particles do respond to turbulence structures equal to or larger than their size, which should lead to structured motion following the nonrandom statistics of the coarse-grained velocity gradient tensor." The paradox may perhaps be explained in the following way: As argued in the previous section, the smaller eddies, which have the largest vorticity, cannot impart a large angular velocity 

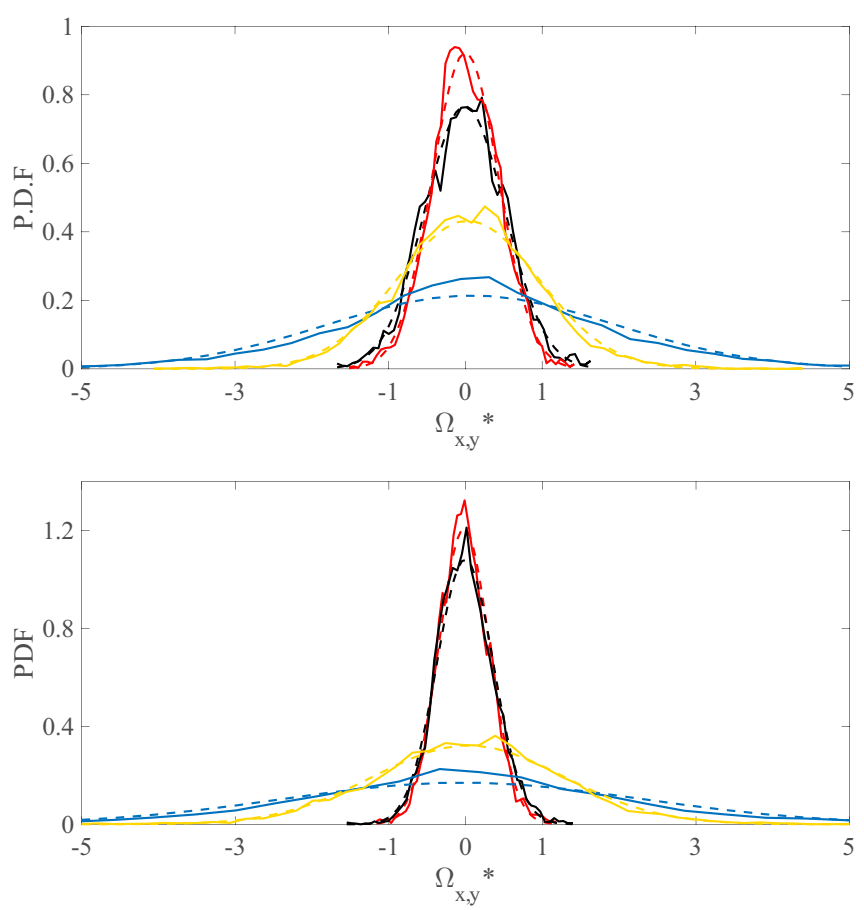

FIG. 2. PDF of the angular velocity in the cross-stream plane $(x, y)$ normalized as in Eq. (11) for $\operatorname{Re}_{p}=80$ (above) and 150. The highest-peaked (red) and second-highest peaked (black) curves are for the shell and the sphere, respectively. The broadest curve (blue) is for the fluid angular velocity (i.e., $\frac{1}{2} \times$ vorticity) averaged over a sphere of radius $d / 2$ (RMS value 3.7179); the other curve (yellow) is for the fluid angular velocity averaged over a sphere of radius $d$ (RMS value 1.8361), both centered at the position of the particle center in the absence of the particle. The dashed lines are Gaussian fits.

to the particle. By the law of large numbers, the accumulation of successive small torques imparted by them will then tend to generate an approximately Gaussian angular velocity distribution. In view of the randomness of the direction of the torque, the angular velocity generated by this process will also necessarily tend to be small, as indeed shown in Fig. 2. Large angular velocities can only be the result of interactions with the less frequent larger eddies, the probability distribution of which is closer to a Gaussian. By these distinct mechanisms, therefore, both the small- and the large-scale eddies conspire to impart to the overall probability distribution of the particle an angular velocity close to a Gaussian.

The PDFs of the dimensionless torque are shown in Fig. 3. Unlike the particle angular velocity, these PDFs are non-Gaussian and exhibit a relatively strong intermittency. The flatness is about 4, which, not coincidentally, given that the torques are due to interaction with the fluid vorticity, is quite close to the flatness of the latter. Qualitatively, these results are similar to those reported in Ref. [7], which deals with a buoyant particle rising in a downward turbulent flow, and which also reports a Gaussian distribution for the angular velocity and significant tails for the angular acceleration. However, the particle Reynolds number in this study was significantly greater than ours and the particle dynamics was strongly affected by vortex shedding. Reference [2] reports a flatness of about 4 for the angular velocity and of about 7 for the angular acceleration. While the relation between the particle size and the integral scale in that study is similar to ours, the two differ substantially in the turbulence level $\left(\operatorname{Re}_{\lambda}=300\right.$ in $\operatorname{Ref}$. [7] versus our $\left.\operatorname{Re}_{\lambda} \sim 30\right)$ and, more importantly, in the fact that the particle of Ref. [2] was neutrally buoyant, free to move and not subjected to a mean flow. 

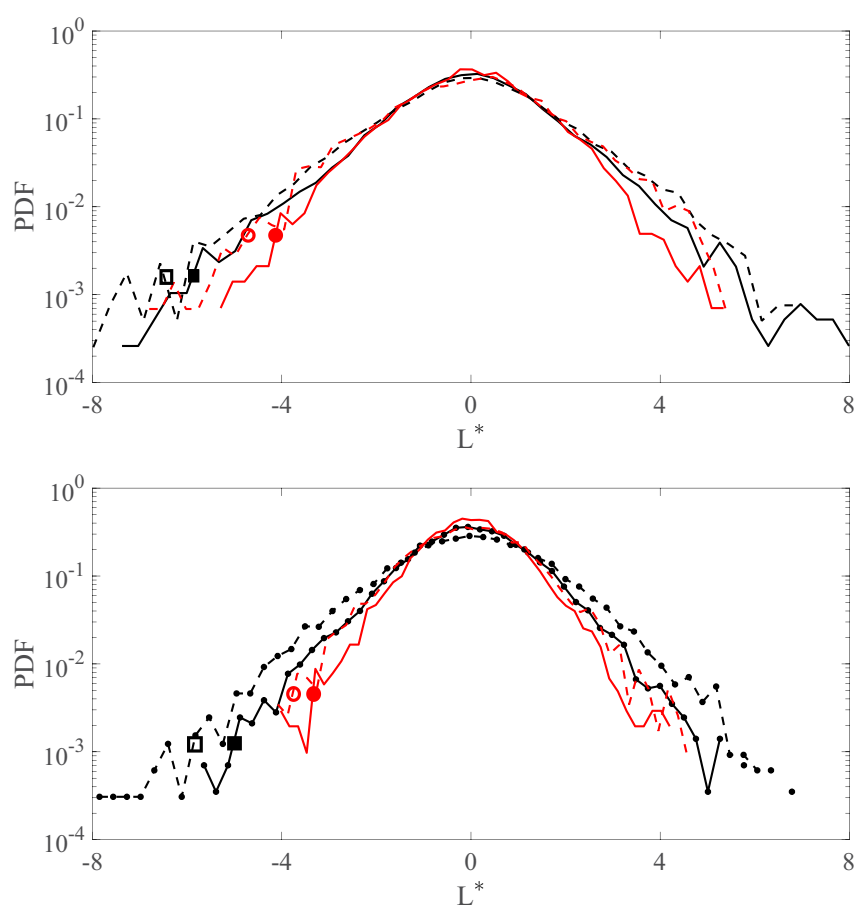

FIG. 3. PDF of the dimensionless torque in the cross-stream plane $(x, y)$ (black lines, squares) and in the direction of the incident flow (red lines, circles) normalized as in Eq. (13) for $\operatorname{Re}_{p}=80$ (above) and 150. The solid lines and solid symbols are for the solid sphere and the dashed lines and open symbols for the spherical shell.

It is also interesting to note that the PDF of the torque acting on the spherical shell has somewhat broader wings than that on the solid sphere. The effect is particularly noticeable for the larger (absolute) values of the torque. A likely explanation is that the greater inertia makes the spherical shell less responsive to the vorticity of the flow, with a consequent larger relative angular velocity and, therefore, larger torques. (An illuminating analogy is the flow past a plate free to move: the faster the plate picks up speed, the faster the drag force decreases.) Another interesting point is the narrower PDF in the direction of the incident flow than in the cross-stream plane, consistently with the smaller values for $L_{z}^{*}$ shown in Table III.

A simple calculation shows that

$$
\frac{\boldsymbol{\Omega} d}{u^{\prime}}=\frac{1}{2 \operatorname{Re}_{p}}\left(\frac{d}{\eta}\right)^{4 / 3} \frac{\boldsymbol{\Omega}^{*}}{u^{\prime} / U} .
$$

With the data of Tables I, II, and III we find results of the order of 0.25 for $\operatorname{Re}_{p}=80$ and 0.18 for $\operatorname{Re}_{p}=150$. In spite of the difference mentioned before between the situations investigated, these magnitudes are comparable to the value $\sim 0.3$ reported in Ref. [2] for neutrally buoyant particles in homogeneous turbulence. The authors point out that these values are consistent with "the rotation that would result from imposing a velocity difference equal to almost $u^{\prime}$ across the diameter of the sphere."

Figure 2 shows that the range of appreciable particle angular velocities extends little beyond the scale of the angular velocity of fluid eddies having the scale of the particle. In the previous section we have provided several considerations on the basis of which this result was to be expected. We reiterate here the importance of the particle rotational inertia demonstrated by the fact that the RMS 


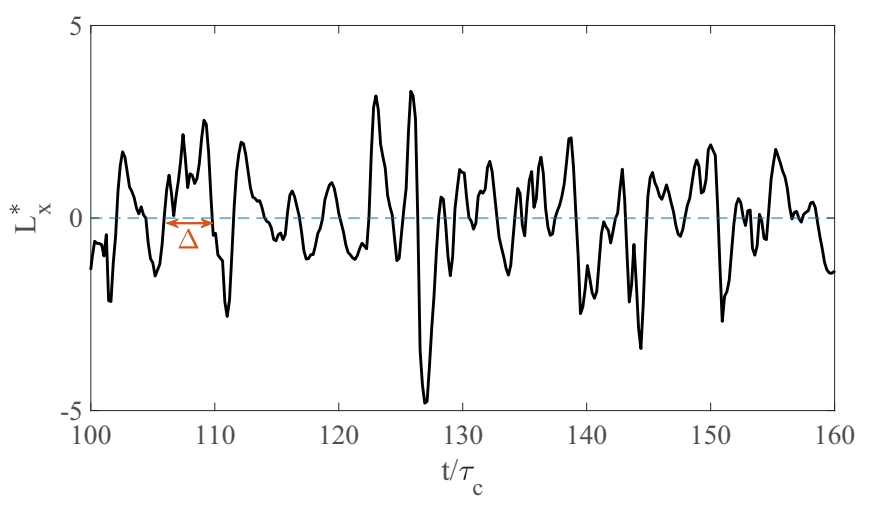

FIG. 4. Time history of the dimensionless torque $L_{x}^{*}$ for the sphere with $\operatorname{Re}_{p}=150$. Time is normalized by the convection time $\tau_{c}=d / U$. The persistence time of the sign of $L_{x}^{*}$ is denoted by $\Delta$.

angular velocity of the shell is smaller than that of the solid sphere and its PDF distribution is narrower and more peaked around zero. Another contributing factor, also mentioned before, is the limited time available for energetic incident turbulent eddies to interact with the particle.

To cast some light on this latter aspect, Fig. 4 shows a portion of the time history of one component of the normalized torque acting on the sphere for $\operatorname{Re}_{p}=150$; time is normalized by the convection time $\tau_{c}$. Rapid sign changes of the torque are evident here, but one also notices a few more extended time intervals, lasting several convection times, in which the torque maintains the same sign.

To investigate the matter quantitatively we consider the sign persistence $\Delta$ of the torque, defined in the figure. The solid lines in the two panels of Fig. 5 show the PDF of this quantity for the two values of $\operatorname{Re}_{p}$. The first data points of the curves correspond to $\Delta / \tau_{c}=0.100$ and 0.187 in the upper and lower panels, respectively, which are the intervals separating two successive data outputs of the simulations. The lack of smoothness displayed by these results is due to the strong intensity of the turbulence the effects of which have not been completely removed by the limited averaging over seven realizations used in this work. The dashed lines show the PDFs of the sign persistence of the cross-stream component of the fluid vorticity, in the absence of the particle, averaged over volumes with radii $d / 4$ (solid square), $d / 2$ (solid circle), $d$ (open square), and $1.5 d$ (open circle). Of course the turbulent eddies will interact with the particle and considering their characteristics disregarding this interaction as we do here requires some justification, which is presented in the Appendix. Briefly, although appeal to the rapid distortion theory of turbulence is only marginally justified even for $\operatorname{Re}_{p}=150$, this theory suggests that incident eddies will retain their sign while interacting with the particle.

These figures present several elements of interest. In the first place, for $\Delta / \tau_{c}$ greater than about $1-2$, it is seen that the sign persistence for the fluid angular velocity agrees with what would be expected on the basis of Eq. (7): the intrinsic timescale of eddies increases with the eddy size, which would lead to a longer sign persistence. (Smaller eddies can also have a longer persistence, but with a low probability as shown in the figure.) The ordering is reversed for small values of $\Delta$, as the lifetime $\tau_{\ell}$ of eddies of scale $\ell$ decreases with $\ell$ as shown by Eq. (7) (and a short persistence of larger eddies is unlikely).

If the sign persistence of the torque is dependent on the sign persistence of the eddies, one would expect the torque to be generated by eddies which, in the absence of the particle, would have a longer lifetime than the torque itself, as these would be damped by the interaction with the particle. We see that, for $\Delta / \tau_{c}$ small $\left(\Delta / \tau_{c}<0.5\right.$ for $\operatorname{Re}_{p}=80$ and $\Delta / \tau_{c}<1$ for $\left.\operatorname{Re}_{p}=150\right)$ eddies with a sign persistence longer than that of the particle angular velocity have a radius of about $d / 4$ for $\operatorname{Re}_{p}=80$ which increases to between $d / 2$ and $d$ for $\operatorname{Re}_{p}=150$. These results suggest that the interactions 

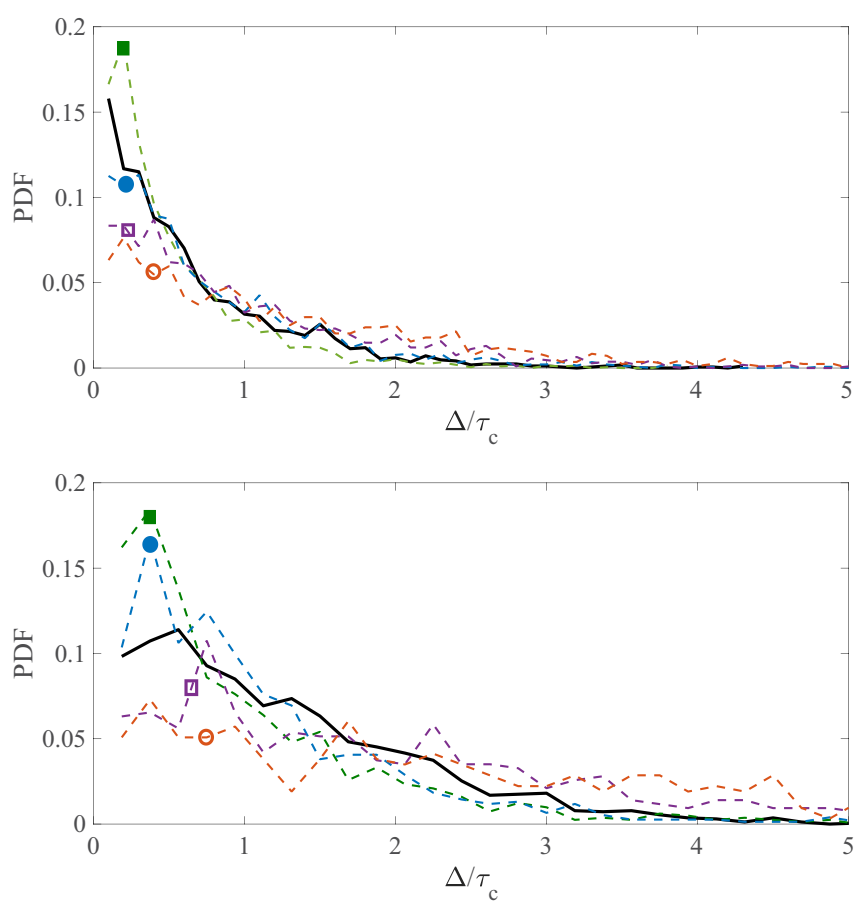

FIG. 5. The solid lines show the PDF's of the sign persistence of the components of the torque in the cross-stream plane acting on the sphere for $\operatorname{Re}_{p}=80$ (upper panel) and $\operatorname{Re}_{p}=150$. The dashed lines are the sign persistence of the cross-stream vorticity in the absence of the particle averaged over spherical volumes with radius $d / 4$ (solid square), $d / 2$ (solid circle), $d$ (open square), and $1.5 d$ (open circle).

giving rise to the most probable sign persistence of the particle angular velocity are with eddies the size of which increases somewhat with the particle Reynolds number, while remaining of a size comparable with that of the particle. The considerations on the "asymptotic eddies" made in Sec. III may explain this result. Eddies with scales between $d / 4$ and $d$ would typically have an angular velocity slightly larger than $O_{\mathrm{fl}}$ but a timescale much shorter than $\tau_{p}$, so that the angular velocity they can impart to the particle will be quite a bit smaller than $O_{\mathrm{fl}}$, as indeed shown by in Fig. 2 .

Figure 2, however, also shows values of the particle angular velocity comparable with $\mathrm{O}_{\mathrm{fl}}$. These larger values must arise from interactions with larger eddies. Indeed, Fig. 5 shows the presence of eddies with a diameter of order $d$ or larger with a sign persistence larger than that of the torque on the particle. As measured on the convective timescale $\tau_{c}$, the sign persistence increases with Reynolds number, probably as a consequence of the estimate Eq. (10) of the scale of eddies lasting a convection time. Another way to look at this feature is to consider the ratio of the characteristic time $\tau_{\ell}$ for an eddy of size $\ell$ to the convection time:

$$
\frac{\tau_{\ell}}{\tau_{c}}=\left(\frac{\ell}{d}\right)^{1 / 3} \frac{\eta}{d} \operatorname{Re}_{p}
$$

With the data of Table I, for the two values of $\operatorname{Re}_{p}$, we find $(\eta / d) \operatorname{Re}_{p}=2.93$ for $\operatorname{Re}_{p}=80$ and $(\eta / d) \operatorname{Re}_{p}=6.81$ for $\operatorname{Re}_{p}=150$. Thus, for the same $\ell / d$, on the scale $\tau_{c}$ eddies at $\operatorname{Re}_{p}=150$ have a longer lifetime than at $\operatorname{Re}_{p}=80$ and, therefore, the torque that they cause also has a longer persistence.

The previous considerations as to the size of eddies responsible for the particle torque are confirmed by an analysis of the autocorrelation time of the torque and of the vorticity averaged over volumes of different sizes, which is shown in Fig. 6. Once again we see that, for $\operatorname{Re}_{p}=80$, the 

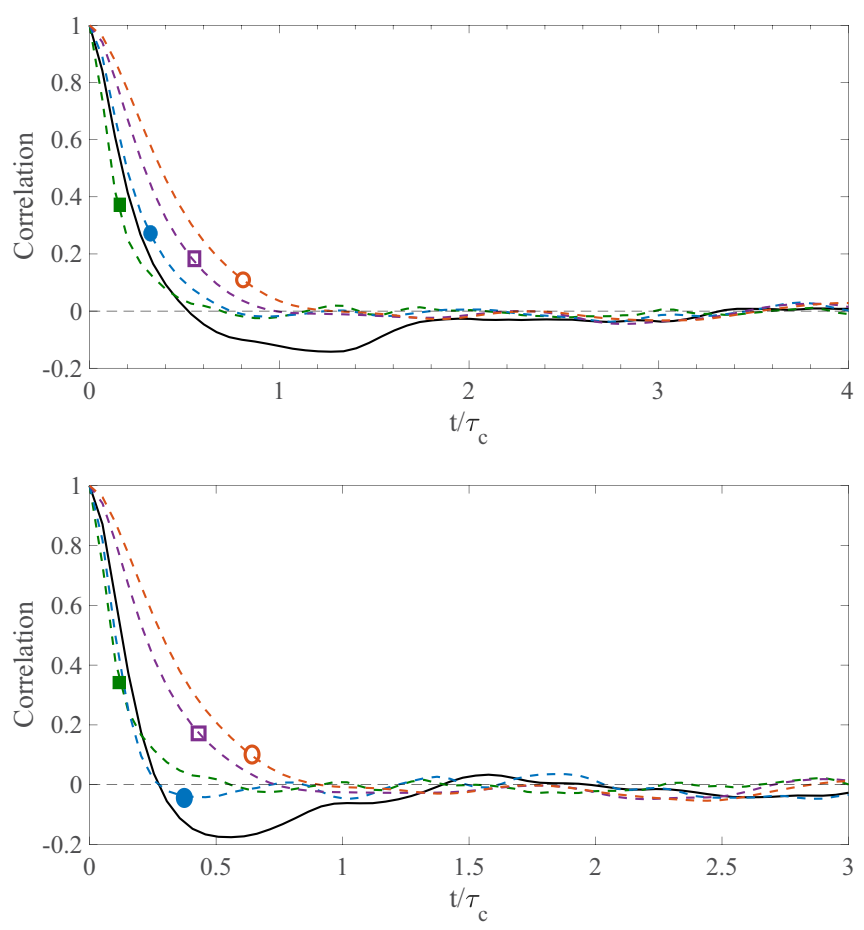

FIG. 6. The solid lines show the autocorrelation of the cross-stream components of the torque acting on the sphere for $\operatorname{Re}_{p}=80$ (upper panel) and $\operatorname{Re}_{p}=150$. The dashed lines are the autocorrelations of the vorticity averaged over spherical volumes with radius $d / 4$ (solid square), $d / 2$ (solid circle), $d$ (open square), and 1.5d (open circle) in the absence of the particle.

torque autocorrelation is intermediate between that of the vorticity averaged over volumes of radii $d / 4$ and $d / 2$ while the scale increases somewhat for $\operatorname{Re}_{p}=150$. It was pointed out earlier after Eq. (8) that eddies with a timescale close to that of the particle are larger than the particle diameter, which would lead one to expect that the autocorrelation of the torque would be closer to that of the larger, rather than the smaller, eddies. Figure 6 shows in fact that the opposite is true. This result suggests that the torque is mostly influenced by eddies smaller than $d$, which are more numerous than larger ones and buffet the particle in greater number as they are convected past it.

For increasing time lags the averaged fluid vorticity becomes only very weakly anti-correlated with itself, in contrast with the torque on the particle, which exhibits a much stronger anticorrelation. This difference is likely a consequence of the particle inertia: the rotation produced by an eddy will cause a torque in the opposite sense whenever another eddy is convected by the incident flow, even if it has the same sign as the previous one (but a different angular velocity), or even if it carries zero vorticity. As $\mathrm{Re}_{p}$ increases, the first zero crossing of the torque occurs earlier and earlier, and significantly before a convection time has elapsed, indicative of the effect of smaller eddies quickly swept by the flow.

Figure 7 shows the autocorrelation of the particle angular velocity normalized by the integral timescale. Two features of this figure demonstrate the effect of rotational inertia. In the first place, the autocorrelation of the angular velocity is seen to be much longer that that of the torque shown in Fig. 6 (note that a given value of $t / \tau_{E}$ in Fig. 7 corresponds to a value of $t / \tau_{c}$ smaller by a factor of about 0.7 for for $\operatorname{Re}_{p}=80$ and 0.3 for $\operatorname{Re}_{p}=150$ ). Second, the first zero crossing for the shell occurs later than for the solid sphere because of the longer persistence of the angular velocity associated with a larger moment of inertia. A third feature present in the numerical results, which we do 


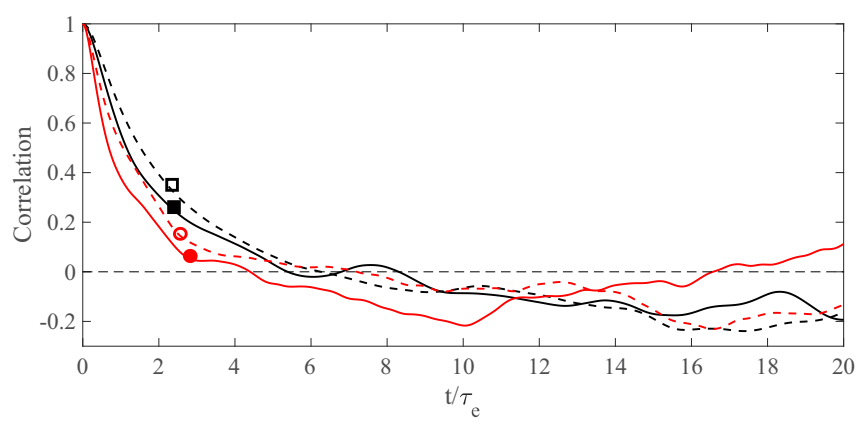

FIG. 7. Autocorrelation of the cross-stream components of the particle angular velocity. The solid lines are for the sphere and the dashed lines for the spherical shell. The squares are for $\operatorname{Re}_{p}=80$ and the circles for $\operatorname{Re}_{p}=150$.

not show for brevity, is the longer sign persistence of the angular velocity for the shell in comparison with the solid sphere. The autocorrelation decays faster at the larger $\operatorname{Re}_{p}$, likely for the same reason mentioned before in connection with the zero-crossing of the autocorrelation of the torque.

\section{RESULTS: FORCES}

Although the force component in the flow direction is dominant, as expected, there are significant forces in the cross stream plane. A possible origin of these components are the velocity fluctuations the action of which may be interpreted as tilting the mean flow incident on the particle as found, for example, in Ref. [25]. Another possibility is vortex shedding. Although the mean-flow Reynolds numbers considered here are much below the threshold for this phenomenon in a uniform steady flow, which is close to 280 [see, e.g., 33], vortex shedding can be induced by the vorticity transported near the particle by the flow as found in Ref. [25]. A third possibility, investigated experimentally in [2] for a neutrally buoyant particle in a homogeneous turbulent field, is a Magnus lift force caused by the interaction of the particle rotation with the incident flow [34-37].

The vortex shedding studied in Refs. [6,7] would tend to impose a relation between the sign of the force induced by the shedding and that of the shed vorticity. Indeed, a vortex shed on one side of the particle imparts to it a force directed toward the opposite side and conservation of angular momentum suggests that the particle would tend to rotate in the direction opposite that of the shed vortex (Fig. 8). With the present choice of axes, a consideration of the signs of forces and torques shows that a positive/negative $F_{x}$ should be associated with a negative/positive $\Omega_{y}$, while $F_{y}$ and $\Omega_{x}$ should have the same sign. Figure 9 shows the PDF of the product $F_{x} \Omega_{y}$ normalized by the product of the RMS values. The PDF is slightly skewed to the left, showing a prevalence of negative values of $F_{x} \Omega_{y}$, which is compatible with the vortex shedding mechanism.

The previous argument suffers from the weakness that, when the particle sheds the vortex, it might already be rotating in the "wrong" direction due to prior encounters with turbulent eddies. In this case vortex shedding would retard the rotation but not necessarily be strong enough to reverse it. To strengthen the previous conclusion it is therefore useful to consider the hydrodynamic torque acting on the particle to which, after all, the acquisition of rotation in the "right" direction after the vortex shedding event would be due. It is clear that the mutual sign relation of $F_{x, y}$ and $L_{x, y}$ should be the same as that between $F_{x, y}$ and $\Omega_{x, y}$ mentioned before. The two panels in Fig. 10 show scatter plots of $L_{y}^{*}$ versus $F_{x}^{*}$ and $L_{x}^{*}$ versus $F_{y} *$ for a single realization with $\operatorname{Re}_{p}=150$; here the dimensionless force components are defined by

$$
\mathbf{F}^{*}=\frac{\mathbf{F}}{\frac{\pi}{8} d^{2} \rho U^{2}},
$$




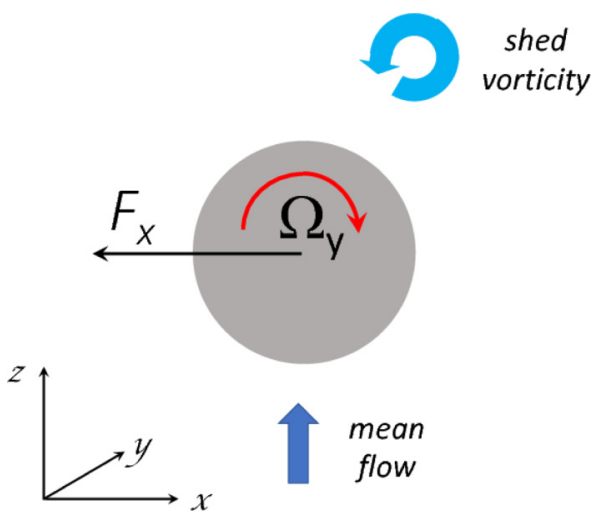

FIG. 8. The shedding of counterclockwise (negative) vorticity on the right imparts to the particle a (negative) reaction force $F_{x}$ to the left and, by conservation of angular momentum, a clockwise (positive) rotation $\Omega_{y}$. As a consequence, this process is associated with a negative value of the products $F_{x} \Omega_{y}$ and $F_{x} L_{y}$.

while $\mathbf{L}^{*}$ is as defined in Eq. (13) above. Both figures show a significant amount of scatter, but a general trend compatible with the sign considerations just described is clear. This conclusion can be reinforced by a consideration of Fig. 11, which shows the PDF of the product $F_{x} L_{y}$ normalized by the respective RMS values for all seven simulations with $\operatorname{Re}_{p}=150$. Here, the bias toward opposite signs is even clearer than in Fig. 9. Numerous instances of vortex shedding can be seen in visualizations of the vorticity distribution. An example is shown in the sequence of Fig. 12 and the movie clip from which this sequence has been extracted is available as Supplemental Material [38].

Plots analogous to that of Fig. 9 for $\operatorname{Re}_{p}=80$ give PDFs that are very nearly symmetric about zero. In this case the turbulence intensity is very large (close to $100 \%$ in fact, as shown in Table I) and force components in the cross-stream directions are mostly due to the rapidly changing direction of the incident flow rather than to vortex shedding (weakened by the smaller incident velocity) or Magnus mechanisms.

The Magnus force on a stationary particle subjected to an incident flow with velocity $\mathbf{V}$ and rotating with angular velocity $\boldsymbol{\Omega}$ is given by [see, e.g., 2,35,39] $\mathbf{F}=C_{L} \rho v \mathbf{V} \times \boldsymbol{\Omega}$, with $C_{L}$ a numerical coefficient of order 1 (Ref. [36] reports $C_{L} \simeq 0.55$, Ref. [35] $C_{L}=\frac{1}{2}$ ) and $v=\frac{1}{6} \pi d^{3}$ the particle volume. Upon using this formula with $V_{z}=U+u_{z}^{\prime}, V_{x, y}=u_{x, y}^{\prime}$ (in which $U$ is the imposed

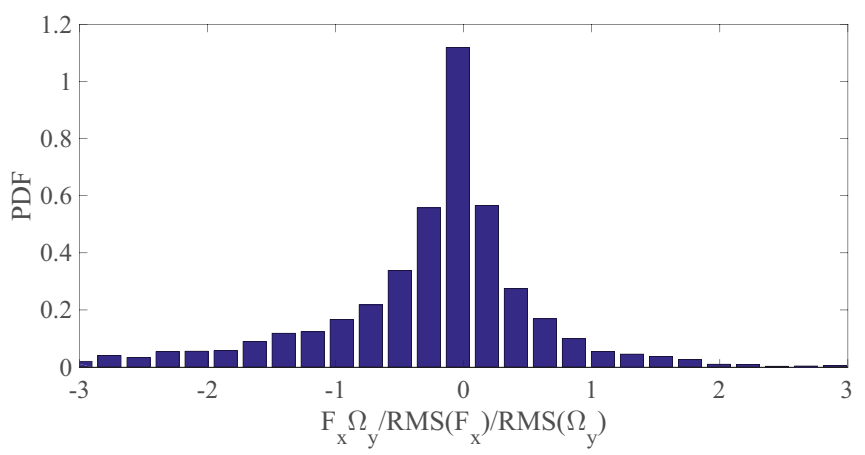

FIG. 9. PDF of the normalized product $F_{x} \Omega_{y}$ for the sphere with $\operatorname{Re}_{p}=150$. The slight bias toward negative values is compatible with a cross-stream force component due to vortex shedding. 

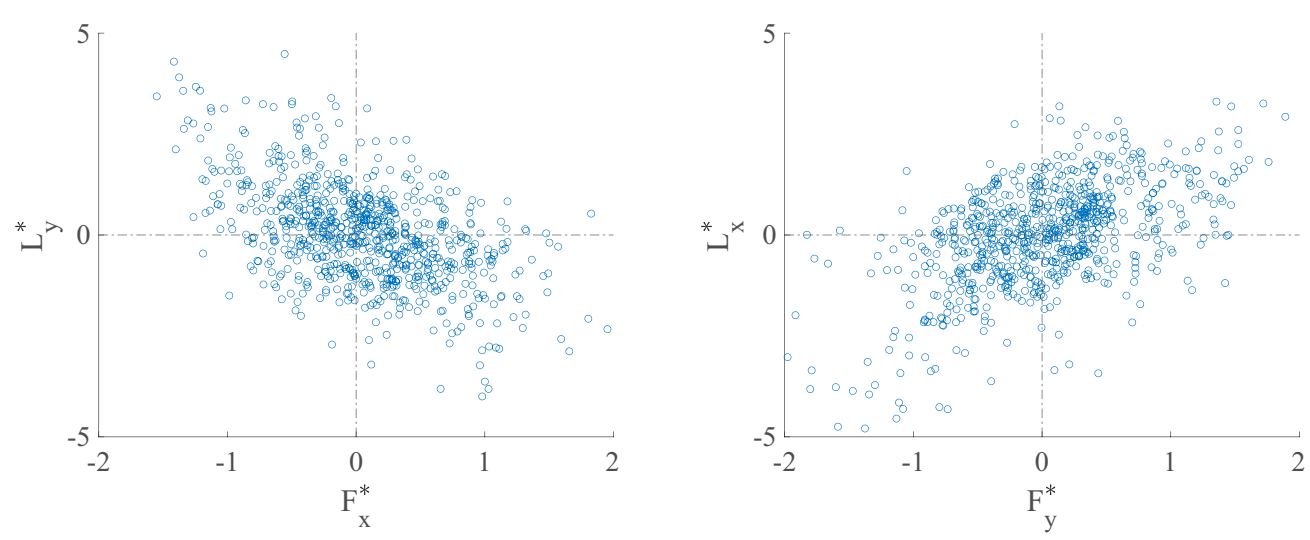

FIG. 10. Scatter plot of $L_{y}^{*}$ vs. $F_{x}^{*}$ (left) and $L_{x}^{*}$ vs. $F_{y}^{*}$ for the sphere with $\operatorname{Re}_{p}=150$ normalized as in Eqs. (13) and (18), respectively. In spite of the large scatter, a trend compatible with vortex shedding from the particle is clearly visible.

mean flow and $u_{x y z}^{\prime}$ are the fluctuating velocity components) we find

$$
F_{x}=C_{L} \rho v\left[\Omega_{z} u_{y}^{\prime}-\Omega_{y}\left(U+u_{z}^{\prime}\right)\right], \quad F_{y}=C_{L} \rho v\left[\Omega_{x}\left(U+u_{z}^{\prime}\right)-\Omega_{z} u_{x}^{\prime}\right] .
$$

In the present situation, at least for $\operatorname{Re}_{p}=150, U$ (which is positive) is dominant and therefore we should expect that, to a very rough approximation,

$$
F_{x} \simeq-C_{L} \rho v \Omega_{y} U, \quad F_{y} \simeq C_{L} \rho v \Omega_{x} U .
$$

The relative sign between force and angular velocity components exhibited by these relations is the same as that due to the vortex shedding phenomenon considered before. Thus, the Magnus mechanism would reinforce vortex shedding to produce a lift force on the sphere, a conclusion also reached in Ref. [2] in spite of the difference in the Reynolds numbers investigated and of the fact that, in that study, the particle was free to move. To gain some insight into the relative importance of the two mechanisms we can study the magnitude of the forces Eq. (20). By nondimensionalizing them according to Eq. (18), we find

$$
F_{x}^{*} \simeq-\frac{2}{3} \frac{1}{\operatorname{Re}_{p}}\left(\frac{d}{\eta}\right)^{4 / 3} C_{L} \Omega_{y}^{*}, \quad F_{y}^{*} \simeq \frac{2}{3} \frac{1}{\operatorname{Re}_{p}}\left(\frac{d}{\eta}\right)^{4 / 3} C_{L} \Omega_{x}^{*} .
$$

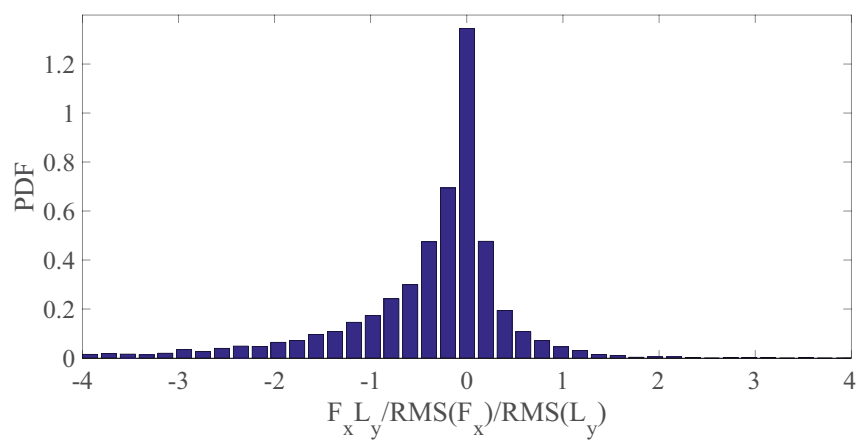

FIG. 11. PDF of the product $F_{x} L_{y}$ normalized by the respective RMS values of the two factors for the sphere with $\operatorname{Re}_{p}=150$. The prevalence of negative values is compatible with the effect of vortex shedding in generating the cross-stream force component $F_{x}$. 
(a)

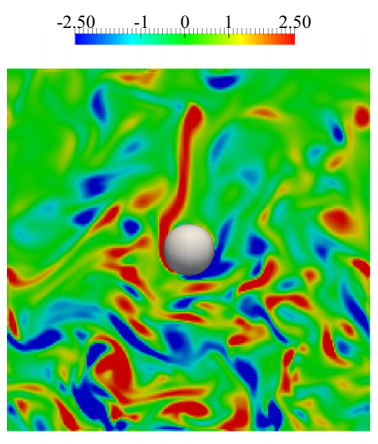

(c)
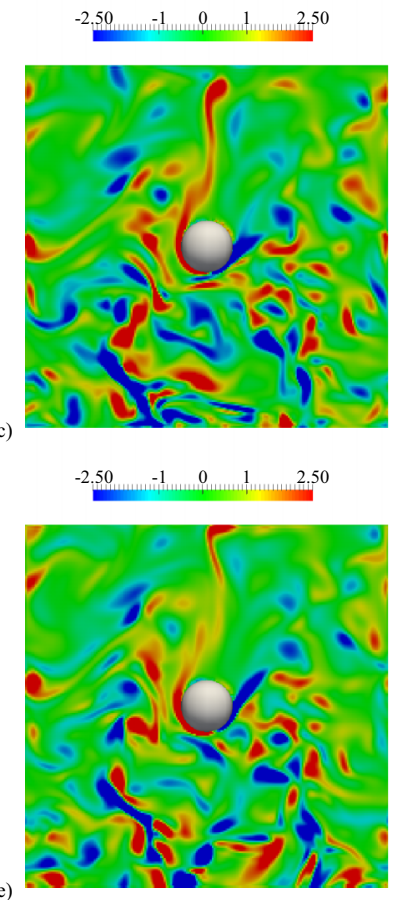

(b)

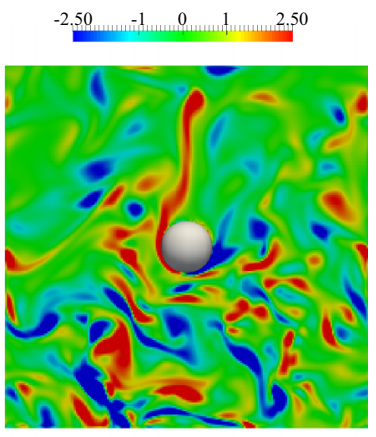

(d)
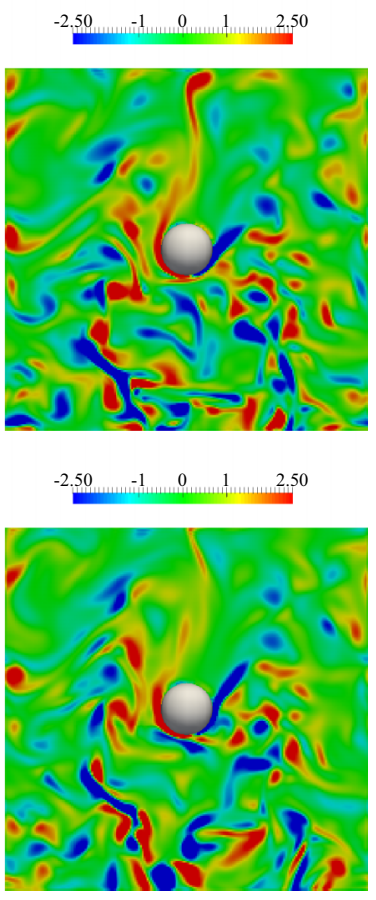

FIG. 12. Six successive images separated by $0.1875 \tau_{c}$ showing an example of the induced shedding of positive (red) vorticity $\Omega_{y}^{*}$ from the sphere for $\operatorname{Re}_{p}=150$. The range of the blue to red color scale is $-2.5 \leqslant$ $\Omega_{y}^{*} \leqslant 2.5$. The mean incident flow is vertically upward in the $z$ direction; the $x$ axis is horizontal to the right and the $y$ axis into the page. This sequence is part of the movie clip available as Supplemental Material [38]; the first image (a) corresponds to approximately $t=31.7 \tau_{c}$ after the start of the movie and the last one (f) to about $t=32.6 \tau_{c}$.

According to Table II the factor multiplying $C_{L} \Omega_{x, y}^{*}$ is approximately 0.274 for $\operatorname{Re}_{p}=150$. Since both $C_{L}$ and $\Omega_{x, y}^{*}$ are smaller than 1 while, from Fig. 10, the normalized cross-stream forces are of order 1 , this result suggests that the Magnus mechanism is somewhat less effective than vortex shedding in generating a cross-stream force on the sphere. This conclusion is not necessarily in contradiction with the results of Ref. [2], because, with a neutrally buoyant sphere free to move, one may expect a much smaller fluid-sphere relative velocity and, therefore, a significantly reduced vortex shedding. For $\operatorname{Re}_{p}=80$ the factor above is 0.686 , but it is difficult to draw definite conclusions due to the very strong turbulence buffeting the sphere in this case. 


\section{SUMMARY AND CONCLUSIONS}

In this paper we have presented results of the fully resolved numerical simulation of turbulent flow $\left(\operatorname{Re}_{\lambda} \simeq 31-36\right)$ past a solid sphere and a spherical shell free to rotate around a fixed center. This situation approximates the behavior of a particle whose relative motion with respect to the fluid is driven by a density difference in the gravitational field. The somewhat artificial condition imposed by the fixity of the particle center is balanced by the advantage of being able to have precise information on the turbulent flow incident on the particle by repeating the same simulation with the particle removed.

By studying the autocorrelation of the torques acting on the particle and the persistence of their sign we have concluded that, at the lower Reynolds number considered, $\operatorname{Re}_{p}=80$, the particle is mostly influenced by turbulent eddies somewhat smaller than itself. As the Reynolds number of the incident flow increases to $\operatorname{Re}_{p}=150$, the scale of the eddies interacting with the particle also increases. The explanation resides in the effects of rotational inertia, as acquiring a significant angular velocity requires exposure of the particle to a fluid torque of the appropriate sign and direction over a certain amount of time. With increasing flow velocity (and, therefore, increasing $\mathrm{Re}_{p}$ ), eddies pass by the particle faster and the required exposure can only take place if the scale of the eddies increases. These results are in qualitative agreement with the experimental observations reported in Ref. [3] (at a higher Reynolds number and with $\operatorname{Re}_{\lambda}=400$ ) according to which the particle is influenced by flow structures with a scale of the order of its diameter. While vortex structures of these scales appear to be most important for the rotational dynamics of the particle, the high frequency of sign reversals of the torque suggests that the effect of these relatively large eddies is modulated by the smaller eddies that they contain.

We have examined the numerical results to detect the presence of cross-stream forces due to vortex shedding and to a Magnus-like mechanism due to the interaction of the particle rotation with the incident flow. Vortex shedding is found to be clearly detectable for $\operatorname{Re}_{p}=150$, in spite of the fact that this Reynolds number is much below that for spontaneous vortex shedding in a uniform flow. The vortex shedding that we find is induced by the interaction with the turbulence transported by the incident flow as was observed an earlier work [25]. A semiquantitative analysis of the results is consistent with the presence of a Magnus mechanism which, however, is found to be less significant than vortex shedding in generating the cross-stream force.

\section{ACKNOWLEDGMENTS}

The authors express their gratitude to two anonymous referees for their insightful comments. Each simulation was run on a single GPU at the Maryland Advanced Research Computing Center. This work has been supported by the National Science Foundation under Grant No. CBET 1335965.

\section{APPENDIX: PERSISTENCE OF THE SIGN OF VORTICITY}

According to the rapid distortion theory of turbulence [see, e.g., Ref. 29], the vorticity transported by a mean flow $\mathbf{V}$ satisfies the approximate relation

$$
\frac{d \omega}{d t}=(\omega \cdot \nabla) \mathbf{V}
$$

in which the convective derivative in the left-hand side is with the velocity $\mathbf{V}$. We approximate the mean incident flow $\mathbf{V}$ as a potential flow, which is reasonable upstream of the separation region, so that

$$
\mathbf{V}=U \mathbf{k}+\nabla\left[U z\left(1+\frac{d^{3}}{16 r^{3}}\right)\right]
$$


in which $\mathbf{k}$ is the unit vector in the direction $z$ of the incident flow, $U$ is the magnitude of the mean incident flow upstream of the sphere and $r=\sqrt{x^{2}+y^{2}+z^{2}}$. If we focus on $\omega_{x}$, then we need to calculate the derivatives of $V_{x}$, which are

$$
\begin{gathered}
\frac{\partial V_{x}}{\partial x}=-\frac{3}{16} \frac{U d^{3} z}{r^{5}}\left(1-5 \frac{x^{2}}{r^{2}}\right) \simeq-\frac{3}{16} \frac{U d^{3} z}{|z|^{5}}, \\
\frac{\partial V_{x}}{\partial y}=\frac{15}{16} \frac{U d^{3} x y z}{r^{7}} \simeq 0 \\
\frac{\partial V_{x}}{\partial z}=-\frac{3}{16} \frac{U d^{3} x}{r^{5}}\left(1-5 \frac{z^{2}}{r^{2}}\right) \simeq 0,
\end{gathered}
$$

where in the last steps we have made the near-axis approximation $|x, y| \ll|z|$. With these results Eq. (A1) gives, approximately,

$$
\frac{d \omega_{x}}{d t} \simeq-\frac{3}{16} \frac{U d^{3} z}{|z|^{5}} \omega_{x} .
$$

This expression shows that, upstream of the sphere (where $z<0$ ), the incident $\omega_{x}$-vorticity increases as the fluid particles approach the sphere. Since, upstream of the sphere, $d \omega_{x} / d t$ and $\omega_{x}$ have the same sign, it is evident that $\omega_{x}$ cannot change sign as the eddy approaches the sphere.

As for the applicability of the rapid distortion theory we can consider the magnitude of

$$
\tau_{E}\left|\frac{d V_{x}}{d x}\right| \simeq \frac{3}{16} \tau_{E} \frac{U d^{3}}{z^{4}}=\frac{3}{16} \operatorname{Re}_{p} \frac{\tau_{E} v}{d^{2}} \frac{d^{4}}{z^{4}} .
$$

Near the sphere, where $z \sim d$, with the data shown in Table II, this quantity is found to be somewhat less than 1 , which puts into question the exact applicability of the rapid distortion theory. Nevertheless, the result is suggestive of the character of the interaction of the eddies with the sphere which appear unlikely to be so strong as to flip the sign of the incident vorticity. Another indication of a certain robustness of the eddy properties as they interact with the particle is given by the near equality of the flatness of the vorticity and of the particle torque noted in Sec. IV.

[1] R. Zimmermann, Y. Gasteuil, M. Bourgoin, R. Volk, A. Pumir, and J.-F. Pinton, Tracking the dynamics of translation and absolute orientation of a sphere in a turbulent flow, Rev. Sci. Instrum. 82, 033906 (2011).

[2] R. Zimmermann, Y. Gasteuil, M. Bourgoin, R. Volk, A. Pumir, and J.-F. Pinton, Rotational Intermittency and Turbulence Induced Lift Experienced by Large Particles in a Turbulent Flow, Phys. Rev. Lett. 106, 154501 (2011).

[3] S. Klein, M. Gibert, A. Bérut, and E. Bodenschatz, Simultaneous 3D measurement of the translation and rotation of finite-size particles and the flow field in a fully developed turbulent water flow, Meas. Sci. Technol. 24, 024006 (2013).

[4] G. Bellani, M. L. Byron, A. G. Collignon, C. R. Meyer, and E. A. Variano, Shape effects on turbulent modulation by large nearly neutrally buoyant particles, J. Fluid Mech. 712, 41 (2012).

[5] M. Byron, J. Einarsson, K. Gustavsson, G. Voth, B. Mehlig, and E. A. Variano, Shape-dependence of particle rotation in isotropic turbulence, Phys. Fluids 27, 035101 (2015).

[6] V. Mathai, V. N. Prakash, J. Brons, C. Sun, and D. Lohse, Wake-Driven Dynamics of Finite-Sized Buoyant Spheres in Turbulence, Phys. Rev. Lett. 115, 124501 (2015).

[7] V. Mathai, M. W. M. Neut, E. P. van der Poel, and C. Sun, Translational and rotational dynamics of a large buoyant sphere in turbulence, Exp. Fluids 57, 51 (2016).

[8] A. D. Bordoloi and E. A. Variano, Rotational kinematics of large cylindrical particles in turbulence, J. Fluid Mech. 815, 199 (2017). 
[9] N. Pujara, T. B. Oehmke, A. D. Bordoloi, and E. A. Variano, Rotations of large inertial cubes, cuboids, cones, and cylinders in turbulence, Phys. Rev. Fluids 3, 054605 (2018).

[10] R. Glowinski, T.-W. Pan, T. I. Hesla, D. D. Joseph, and J. Periaux, A fictitious domain approach to the direct numerical simulation of incompressible viscous flow past moving rigid bodies: Application to particulate flow, J. Comput. Phys. 169, 363 (2001).

[11] M. Uhlmann, An immersed boundary method with direct forcing for the simulation of particulate flows, J. Comput. Phys. 209, 448 (2005).

[12] M. Uhlmann, Interface-resolved direct numerical simulation of vertical particulate channel flow in the turbulent regime, Phys. Fluids 20, 053305 (2008).

[13] M. Mehrabadi, S. Tenneti, R. Garg, and S. Subramaniam, Pseudo-turbulent gas-phase velocity fluctuations in homogeneous gas-solid flow: fixed particle assemblies and freely evolving suspensions, J. Fluid Mech. 770, 210 (2015).

[14] F. Picano, W.-P. Breugem, and L. Brandt, Turbulent channel flow of dense suspensions of neutrally buoyant spheres, J. Fluid Mech. 764, 463 (2015).

[15] A. Noorani, G. Sardina, L. Brandt, and P. Schlatter, Particle transport in turbulent curved pipe flow, J. Fluid Mech. 793, 248 (2016).

[16] S. Balachandar and J. K. Eaton, Turbulent dispersed multiphase flow, Annu. Rev. Fluid Mech. 42, 111 (2010).

[17] E. Calzavarini, R. Volk, E. Lévêque, J.-F. Pinton, and F. Toschi, Impact of trailing wake drag on the statistical properties and dynamics of finite-sized particle in turbulence, Physica D 241, 237 (2012).

[18] A. Naso and A. Prosperetti, The interaction between a solid particle and a turbulent flow, New J. Phys. 12, 033040 (2010).

[19] S. Parsa and G. A. Voth, Inertial Range Scaling in Rotations of Long Rods in Turbulence, Phys. Rev. Lett. 112, 024501 (2014).

[20] K. Gudmundsson and A. Prosperetti, Improved procedure for the computation of Lamb's coefficients in the Physalis method for particle simulation, J. Comput. Phys. 234, 44 (2013).

[21] A. J. Sierakowski and A. Prosperetti, Resolved-particle simulation by the Physalis method: Enhancements and new capabilities, J. Comput. Phys. 309, 164 (2016).

[22] A. J. Sierakowski, GPU-centric resolved-particle disperse two-phase flow simulation using the Physalis method, Phys. Commun. 207, 24 (2016).

[23] H. Lamb, Hydrodynamics, 6th ed. (Dover, New York, NY, 1932).

[24] S. Kim and S. J. Karrila, Microhydrodynamics: Principles and Selected Applications (ButterworthHeinemann, Boston, MA, 1991).

[25] L. Botto and A. Prosperetti, A fully resolved numerical simulation of turbulent flow past one or several particles, Phys. Fluids 24, 013303 (2012).

[26] S. Lundgren, Linearly forced isotropic turbulence, in Annual Research Briefs (Stanford University Press, Stanford, CA, 2003), pp. 461-473.

[27] C. Rosales and C. Meneveau, Linear forcing in numerical simulations of isotropic turbulence: Physical space implementations and convergence properties, Phys. Fluids 17, 095106 (2005).

[28] P. L. Carroll and G. Blanquart, A proposed modification to Lundgren's physical space velociy forcing method for isotropic turbulence, Phys. Fluids 25, 105114 (2013).

[29] S. B. Pope, Turbulent Flows (Cambridge University Press, Cambridge, UK, 2000).

[30] F. W. Roos and W. W. Willmarth, Some experimental results on sphere and disk drag, AIAA J. 9, 285 (1971).

[31] R. Clift, J. R. Grace, and M. E. Weber, Bubbles, Drops and Particles (Academic Press, New York, NY, 1978), reprinted by Dover.

[32] C. Crowe, M. Sommerfield, and Y. Tsuji, Multiphase Flows with Droplets and Particles (CRC Press, Boca Raton, FL, 1998).

[33] R. Natarajan and A. Acrivos, The instability of the steady flow past spheres and disks, J. Fluid Mech. 254, 323 (1993).

[34] R. Rubinov and J. B. Keller, The transverse force on a spinning sphere moving in a viscous fluid, J. Fluid Mech. 11, 447 (1961). 
[35] T. R. Auton, The lift force exerted on a spherical body in rotational flow, J. Fluid Mech. 183, 199 (1987).

[36] P. Bagchi and S. Balachandar, Effect of free rotation on the motion of a solid sphere in linear shear flow at moderate Re, Phys. Fluids 14, 2719 (2002).

[37] J. J. Bluemink, D. Lohse, A. Prosperetti, and L. van Wijngaarden, Drag and lift forces on particles in a rotating flow, J. Fluid Mech. 643, 1 (2010).

[38] See Supplemental Material at http://link.aps.org/supplemental/10.1103/PhysRevFluids.4.064304 for a sequence showing the interaction of the particle with the incident vorticity for $\operatorname{Re}_{p}=150$.

[39] T. R. Auton, J. C. R. Hunt, and M. Prudhomme, The force exerted on a body in inviscid unsteady nonuniform flow, J. Fluid Mech. 197, 241 (1988). 